\title{
T2-Dark Restricted Diffusion
}

\author{
D.M. Mandell, D.J. Mikulis, T.R. Kiehl, T. Krings
}

Can J Neurol Sci. 2012; 39: 664-666

Magnetic resonance signal intensity on diffusion-weighted images (DWI) is influenced by proton density (PD), transverse relaxation time (T2), apparent diffusion coefficient (ADC), bvalue, and echo time (TE) according to the equation ${ }^{1}$ :

Signal Intensity ${ }_{\text {DWI }}=k$ (Proton Density $)\left(\mathrm{e}^{-\mathrm{TE} / \mathrm{T} 2} \mathrm{e}^{-(\mathrm{b} \text { value)(ADC)})}\right.$

where $\mathrm{k}$ is a constant, and "b-value" denotes sensitivity of the MR pulse sequence to diffusion effects. Transverse relaxation time and ADC have opposing influences on DWI signal intensity: hyperintensity on T2-weighted images (that is, long T2) results in hyperintensity on DWI, whereas increased ADC results in hypointensity on DWI. Interpretation of clinical studies requires an understanding of these relationships. For example, hyperintensity on a diffusion-weighted image may arise from the decreased ADC of an acute infarct, but also from the increased $\mathrm{T} 2$ of a tumor or other lesion.

There are three well-known interactions between T2, ADC, and DWI. These three patterns, termed "T2 shine through", "T2 blackout", and "T2 washout", are illustrated schematically in Figure 1. Reviewing the topic, Hiwatashi et $\mathrm{al}^{1}$ described a potential fourth interaction - isointensity on diffusion-weighted images caused by a balance of hypointensity on T2-weighted images and decreased apparent diffusion coefficient - but there is only very limited literature ${ }^{2}$ on this pattern. This fourth pattern ("*" in Figure 1) is important as it may yield misleading diffusion-weighted images that are "false negative" for acute ischemic injury. We report a case which demonstrates isointensity on diffusion-weighted images caused by a balance of hypointensity on T2-weighted images and decreased ADC.

\section{CASe}

A 49-year-old woman, with a past medical history of migraine headaches and diabetes mellitus type 2, presented to an emergency department after a four day history of bumping into walls. On visual field testing by direct confrontation, she had a left homonymous hemianopsia (later confirmed with a Humphrey visual field test). The neurological examination was otherwise essentially unremarkable. Magnetic resonance imaging and computed tomogram (CT) angiography demonstrated an arteriovenous malformation (AVM) in the right occipital lobe, with no evidence of hemorrhage or infarction. Catheter angiography (Figure 2) showed a nidus-type AVM, supplied by a mildly enlarged right parieto-occipital branch of the right posterior cerebral artery with indirect leptomeningeal recruitment from the calcarine branch of the posterior cerebral artery, supply from right middle cerebral artery branches, some recruitment of right anterior cerebral artery branches, and

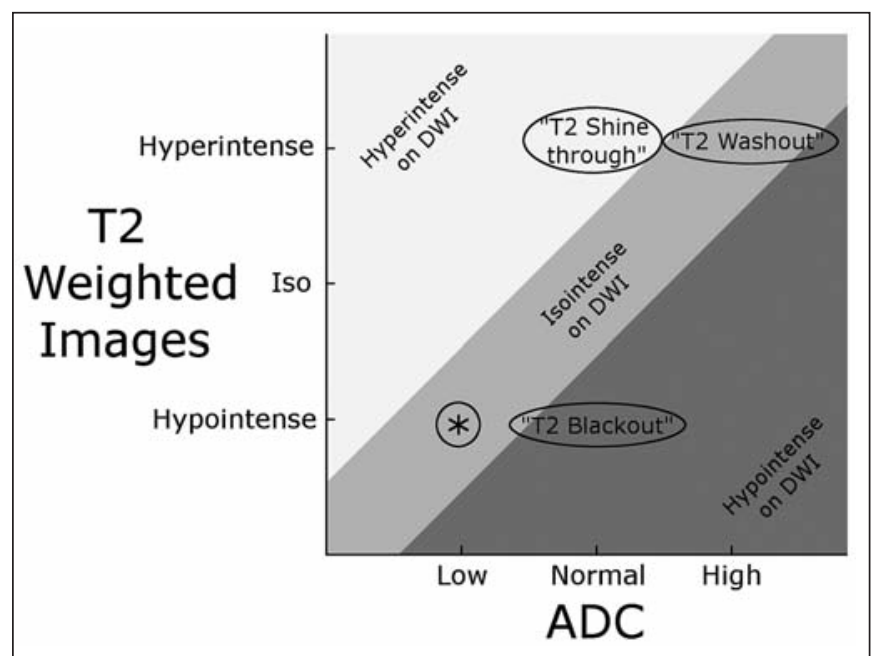

Figure 1: Schematic illustrating the interactions between T2, apparent diffusion coefficient, and DWI. "T2 blackout" refers to hypointensity on DWI from a combination of normal ADC and hypointensity on T2weighted images. Conversely, "T2 shine through" refers to hyperintensity on DWI from a combination of normal ADC and hyperintensity on T2-weighted images. "T2 washout" refers to isointensity on DWI from a combination of hyperintensity on T2weighted images and increased ADC. Misleading isointensity on DWI, due to decreased $A D C$ and shortened $T 2$ relaxation, and described in this report, is shown as well (*).

perinidal neoangiogenesis. Venous drainage was via superficial cortical veins into the superior sagittal sinus and right transverse sinus. There was no evidence of venous outflow stenosis or thrombosis, and no evidence of venous congestion. The patient was discharged home for follow-up at an outpatient neurovascular disease clinic.

At one-month follow-up, the patient reported a persisting visual field defect. Repeat MR imaging (Figure 3) demonstrated a large region of decreased ADC in the right parietal, occipital, and temporal lobes, predominantly in the white matter, consistent with restricted diffusion. Diffusion-weighted

From the Division of Neuroradiology (DMM, DJM, TK) and Department of Pathology (TRK), University Health Network and the University of Toronto, Toronto, Ontario, Canada.

Received November 28, 2011. Final Revisions Submitted May 3, 2012. Correspondence to: Daniel Mandell, Department of Medical Imaging, Toronto Western Hospital, Room 3MC-426, 399 Bathurst Street, Toronto, Ontario, M5T 2S8, Canada. Email: danny.mandell@uhn.ca. 


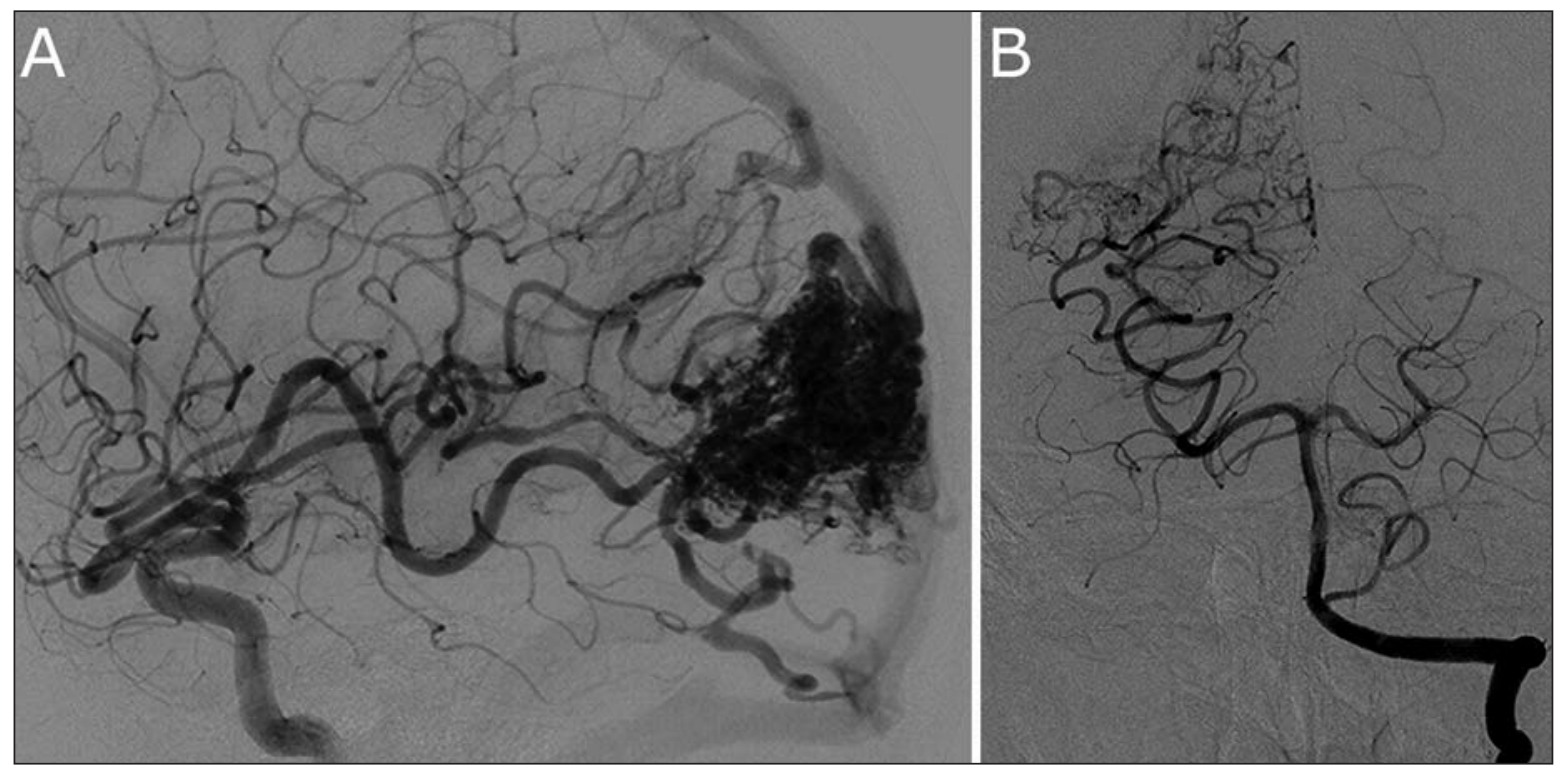

Figure 2: Catheter angiography showing right internal carotid artery $(A)$ and left vertebral artery (B) injections. There is a nidustype AVM supplied predominantly by right middle and posterior cerebral artery branches, and draining via cortical veins into the superior sagittal sinus and right transverse sinus. There is perinidal neoangiogenesis.

(isotropic) images demonstrated relatively isointense signal throughout most of this region. T2-weighted images showed hypointensity in the large region of white matter that had demonstrated a combination of deceased ADC and isointensity on diffusion-weighted images. There was no evidence of abnormal blooming on a $\mathrm{T} 2 *$-weighted gradient echo sequence. An electroencephalogram was performed. This demonstrated intermittent abnormal spikes over the right parietal and occipital lobes, but no activity suggestive of status epilepticus.
At two-month follow-up, the patient had a third MR imaging exam (Figure 4). This demonstrated normalization of the ADC maps. The previous hypointensity on T2-weighted images had converted to hyperintensity, and there was corresponding T2 shine through on diffusion-weighted images. The AVM was then resected. Histopathological evaluation revealed variably thick walled vessels, typical of an AVM, with numerous neutrophils adhered to the endothelial surface, and surrounding the AVM, vacuolated gliotic white matter with reactive astrocytes, sheets of macrophages, and occasional lymphocytes.
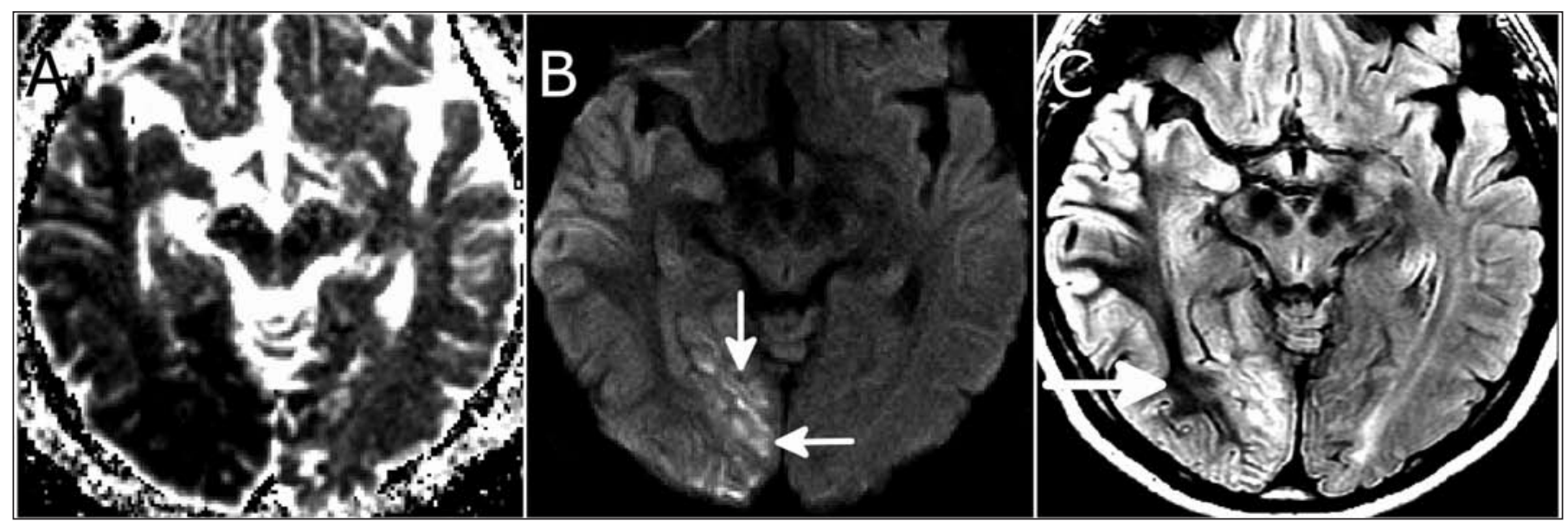

Figure 3: MR imaging of the brain, using a 3 Tesla MR system with 8-channel phased array head coil, at one-month follow-up visit. ADC map (A) demonstrates a large region of low signal intensity in the right occipital and temporal lobes, predominantly in the white matter, consistent with restricted diffusion. Diffusion-weighted image $(b$-value $=1000 \mathrm{~s} / \mathrm{mm} 2)$. (B) shows relatively isointense signal throughout most of this region, with hyperintensity limited to a small portion of the right occipital lobe (arrow). T2-weighted image (C) show hypointensity (arrow) in the large region of white matter that demonstrated a combination of decreased ADC and isointensity on DWI. Note, the AVM nidus is not shown in the slice in this figure. 


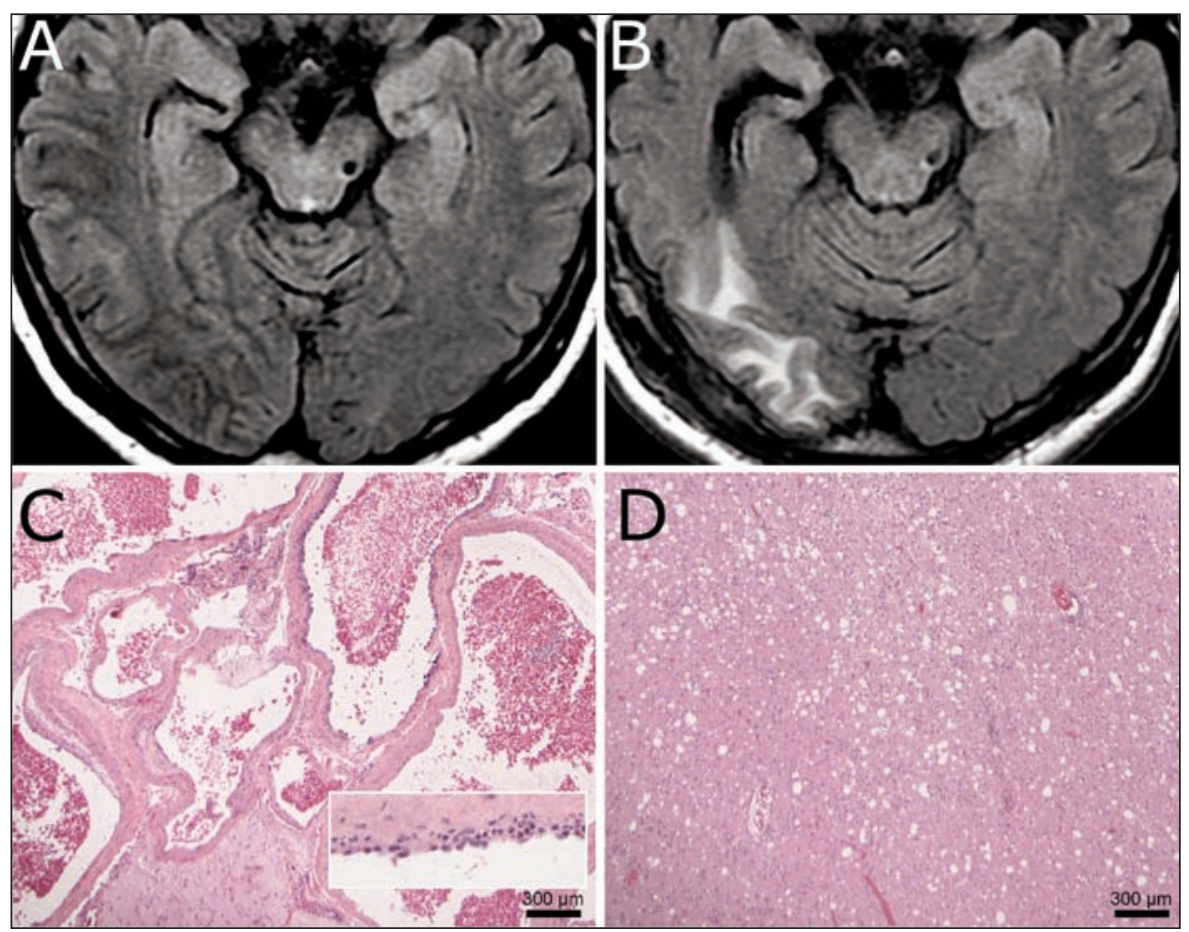

Figure 4: MR imaging of the brain, using a 1.5 Tesla MR system, at presentation (A) and two months later (b). T2-weighted fluid attenuation inversion recovery (FLAIR) images demonstrate development of right occipitotemporal brain atrophy and confluent white matter hyperintensity on the second study. (C) Histopathologic examination showed a typical AVM, characterized by variably-sized blood vessels without internal elastic layer, as well as intervening gliotic brain (lower middle). Frequent marginating neutrophils were present (inset). (D) Adjacent brain showed extensive vacuolation and astrogliosis with large numbers of macrophages and mild lymphocytic inflammation.

\section{DisCUSSION}

We have demonstrated misleading isointensity on diffusionweighted images caused by a balance of hypointensity on T2weighted images and decreased ADC. This interaction is distinct from the previously described T2 shine through, T2 blackout, and T2 washout effects. The pattern is important as it may yield diffusion-weighted images that are false negative for acute ischemic injury.

What is the pathophysiology underlying the combination of hypointensity on T2-weighted images and decreased ADC? Confluent hypointensity in the white matter on T2-weighted image has been reported in hypoxia, cortical ischemia, seizure activity, meningitis, encephalitis, leptomeningeal metastatic disease, and other disorders. In our case, the EEG and histopathology findings argue against seizure activity as an explanation, and we therefore suspect an injury in the hypoxiaischemia category. A potential explanation for the $\mathrm{T} 2$ shortening is disruption of axonal iron transport, with parenchymal deposition of iron yielding a paramagnetic effect. ${ }^{3}$ However, on histological evaluation of animals with hypoxic-ischemic brain injury, ${ }^{4}$ and a human with viral encephalitis and white matter T2 shortening, ${ }^{2}$ there was no evidence of iron deposition in the white matter. Another possibility is accumulation of paramagnetic oxygen species, that is, free radicals. During cerebral ischemia and reperfusion, increased production of free radicals can exceed scavenging capacity. ${ }^{5}$ In the context of an AVM, with perinidal neoangiogenesis, which is potentially a response to arterial steal effect and chronic perinidal hypoperfusion, one might hypothesize that excessive free radical production exists. Lee et $\mathrm{al}^{2}$ noted slightly decreased ADC in patients with subcortical hypointensity on T2-weighted images, and postulated that an underlying paramagnetic substance may account for both signal changes. In our case, progression to gliosis and vacuolization (Figure 4) in the region of prior low ADC might suggest that the ADC reduction was truly due to cytotoxic edema rather than transient free radical accumulation alone.

We have described a case of false negative ischemia on diffusion-weighted images caused by a balance of hypointensity on T2-weighted images and decreased ADC, that is, a pattern of "T2-dark restricted diffusion." This pattern is distinct from the previously described T2 shine through, T2 blackout, and T2 washout effects. The pattern is clinically important as one may falsely interpret such diffusion-weighted images as negative for cytotoxic edema.

\section{REFERENCES}

1. Hiwatashi A, Kinoshita T, Moritani T, et al. Hypointensity on diffusion-weighted MRI of the brain related to T2 shortening and susceptibility effects. AJR Am J Roentgenol. 2003;181:1705-9.

2. Lee JH, Na DG, Choi KH, et al. Subcortical low intensity on MR images of meningitis, viral encephalitis, and leptomeningeal metastasis. AJNR Am J Neuroradiol. 2002;23:535-42.

3. Ida M, Mizunuma K, Hata Y, et al. Subcortical low intensity in early cortical ischemia. AJNR Am J Neuroradiol. 1994;15:1387-93.

4. Palmer C, Menzies SL, Roberts RL, et al. Changes in iron histochemistry after hypoxic-ischemic brain injury in the neonatal rat. J Neurosci Res. 1999;56:60-71.

5. Guo ZH, Li F, Wang WZ, Guo Z-H, Li F, Wang W-Z. The mechanisms of brain ischemic insult and potential protective interventions. Neurosci Bulletin. 2009;25:139-52. 